\title{
The influence of slippage on trapping and reflux limits with peristalsis through an asymmetric channel
}

\author{
Z.M. Gharsseldien ${ }^{\mathrm{a}, \mathrm{b} *}$, Kh.S. Mekheimer ${ }^{\mathrm{b}}$ and A.S. Awad ${ }^{\mathrm{c}}$ \\ ${ }^{a}$ Mathematics Department, Faculty of Arts \& Science, King Saud University, Wadi Adwassir, Saudi Arabia; ${ }^{\mathrm{b}}$ Mathematics Department, \\ Faculty of Science for Men, Al-Azhar University, Cairo, Egypt; ' Mathematics Department, Faculty of Science for Girls, Al-Azhar \\ University, Cairo, Egypt.
}

(Received 4 May 2009; final version received 30 March 2010)

\begin{abstract}
The effects of slip boundary condition on peristaltic transport of incompressible Newtonian viscous fluid in an asymmetric channel is investigated, under the conditions of low Reynolds number and long wavelength. The pumping characteristics, trapping and reflux limits are studied for different values of the dimensionless slip parameter $\beta$.
\end{abstract}

Keywords: peristaltic motion; asymmetric channel; trapping; reflux; slippage

\section{Introduction}

Peristalsis is defined as a wave of relaxation or contraction (expansion) of the walls of a flexible conduit, which pumps the enclosed material inside or outside the conduit (Mekheimer 2003; Mishra and Rao 2003). Peristalsis is now well known to the physiologists to be one of the major mechanisms of fluid transport in many biological systems, e.g. swallowing food through the oesophagus, movement of chyme in the gastrointestinal tract, urine transport from the kidney to the bladder through the ureter, transport of spermatozoa, movement of ova in the fallopian tube, vasomotion of small blood vessels such as venules and capillaries and blood flow in arteries and in many other glandular ducts (Srivastava and Srivastava 1982; El-Shehawey and Husseny 2000; Mekheimer 2003; Mishra and Rao 2003; El-Shehawey et al. 2006; El-Hakeem et al. 2006). There are many industrial applications for peristaltic transport, like blood pumps in a heart-lung machine, and furthermore there are many applications in biomechanical and engineering sciences for peristaltic motion (ElShehawey and Husseny 2000; Mishra and Rao 2003). Peristaltic motion results physiologically from neuron muscular properties of the tubular smooth muscles (Gharsseldien 2003). Recently, some studies have proven that the intra-uterine fluid flow due to myometrial contraction is a peristaltic motion (Eytan et al. 2001; Strandell and Lindhard 2002; Nakai et al. 2003, 2004; Nishino et al. 2005).

Several mathematical and experimental models have been developed to understand the fluid mechanical aspects of peristaltic flows with considerations of the nature of the fluid, the geometry of the channel, propagating waves and inclusion of other physical effects such as magnetic fields and porous media (Fung and Yih 1968; Srivastava and Srivastava 1982; Gharsseldien 2003; Mekheimer 2003; Mishra and Rao 2003; El-Shehawey et al. 2006; Ali and Hayat 2007, 2008; Hayat et al. 2008).

We are aware that the no-slip condition in fluid mechanics means that the fluid velocity matches the velocity of the solid boundary. Nearly 200 years ago Navier proposed in his original paper on linearly viscous fluids a general boundary condition that permits the possibility of slip at a solid boundary (Joshi and Denn 2003). This boundary condition assumes that the tangential velocity of the fluid relative to the solid at a point on its surface is proportional to the tangential stress acting at that point. The constant of proportionality between these two quantities may be termed a coefficient of sliding friction, which is assumed to depend on the nature of the fluid and the solid surface:

$$
U=\mp \eta \tau_{w},
$$

where $U$ is the tangential velocity in the $X$ direction; $\eta$ is a constant of proportionality (the slip parameter); and $\tau_{w}$ is the shear stress at the wall (Rao and Rajagopal 1999; Joshi and Denn 2003; Faltas and Saad 2005).

Recently, some studies that explain the effect of slip boundary conditions on the peristaltic dynamics for different fluids under different conditions have been carried out (El-Shehawey et al. 2006; Ali et al. 2008; Ebaid 2008; Hayat et al. 2008,b; Ellahi 2009). But to the best of our knowledge, no one has attempted to calculate the trapping and reflux limits for a slip flow in an asymmetric channel with peristalsis.

*Corresponding author. Email: gharsseldien_z@yahoo.com 
The aim of this paper is to investigate the influence of the slip boundary conditions on the trapping phenomena and the reflux limits with peristaltic transport in an asymmetric channel under the conditions of low Reynolds number and long wavelength. Therefore, we will consider peristaltic transport in the uterus (transport of intra-uterine fluid flow) as an example of the applications which involve the peristaltic transport technique in an asymmetric channel with the slip condition.

\section{Formulation of the problem and solution}

We consider the motion of incompressible viscous Newtonian fluid in a two-dimensional asymmetric channel. Equations of motion are governed by the Navier-Stokes equations in two dimensions,

$$
\begin{aligned}
& \frac{\partial U}{\partial t}+U \frac{\partial U}{\partial X}+V \frac{\partial U}{\partial Y}=-\frac{1}{\rho} \frac{\partial P}{\partial X}+v \nabla^{2} U, \\
& \frac{\partial V}{\partial t}+U \frac{\partial V}{\partial X}+V \frac{\partial V}{\partial Y}=-\frac{1}{\rho} \frac{\partial P}{\partial Y}+v \nabla^{2} V
\end{aligned}
$$

and the continuity equation

$$
\frac{\partial U}{\partial X}+\frac{\partial V}{\partial Y}=0
$$

where $U$ and $V$ are the fluid velocity components in the $X$ and $Y$ directions in laboratory frame; $\rho$ is the density of the fluid; $P$ is the pressure; $v$ is the kinematic viscosity ( $v=\mu / \rho$, with $\mu$ the coefficient of the viscosity of the fluid); and

$$
\nabla^{2}=\frac{\partial^{2}}{\partial X^{2}}+\frac{\partial^{2}}{\partial Y^{2}}
$$

The channel asymmetry is produced by choosing the peristaltic wave train on the walls to have different amplitudes and phase as shown in Figure 1. Consider the upper and lower channel walls:

$$
\begin{aligned}
& \mathbf{Y}=H_{1}=d_{1}+a_{1} \cos \frac{2 \pi}{\lambda}(x-c t), \\
& \mathbf{Y}=H_{2}=-d_{2}-b_{1} \cos \left[\frac{2 \pi}{\lambda}(x-c t)+\phi\right]
\end{aligned}
$$

where $a_{1}$ and $b_{1}$ are the amplitudes of the waves; $\lambda$ is the wavelength; $\left(d_{1}+d_{2}\right)$ is the width of the channel; and $\phi$ is the phase difference that varies in the range $0 \leq \phi \leq \pi$. Further, $a_{1}, b_{1}, d_{1}, d_{2}$ and $\phi$ satisfy the condition $\bar{a}_{1}^{2}+\bar{b}_{1}^{2}+$ $2 a_{1} b_{1} \cos \phi \leq\left(d_{1}+d_{2}\right)^{2}$.

Now introduce a wave frame $(x, y)$ moving with the velocity $c$ away from the fixed frame $(X, Y)$ by the trans- formations

$$
\begin{aligned}
x & =X-c t, \quad y=Y, \quad u=U-c, \quad v=V, \\
p(x) & =P(X, t),
\end{aligned}
$$

where $u$ and $v$ are the fluid velocity components and $p$ is the pressure in the wave frame of references.

Furthermore, introduce the Reynolds number $R$, the wave number $\delta$, the stream function $\psi$ (where $u=\partial \psi / \partial y$ and $v=-\partial \psi / \partial x$ ) and the following non-dimensional variables:

$$
\begin{gathered}
\bar{x}=\frac{x}{\lambda}, \quad \bar{y}=\frac{y}{d_{1}}, \quad \bar{u}=\frac{u}{c}, \quad \bar{v}=\frac{v}{c \delta}, \quad \delta=\frac{d_{1}}{\lambda}, \\
R=\frac{c d_{1}}{v}, \quad \bar{t}=\frac{c t}{\lambda}, \quad a=\frac{a_{1}}{d_{1}}, \quad b=\frac{b_{1}}{d_{1}}, \quad d=\frac{d_{2}}{d_{1}}, \\
h_{1}=\frac{H_{1}}{d_{1}}, \quad h_{2}=\frac{H_{2}}{d_{1}}, \quad \bar{\psi}=\frac{\psi}{c d_{1}}, \quad \bar{p}=\frac{d_{1}^{2} p}{\mu \lambda c} .
\end{gathered}
$$

Using the transformations (6) and the non-dimensional variables (7) and after eliminating the pressure and dropping the bars, Equations (1)-(3) in terms of the stream function will take the form

$$
\begin{aligned}
& R \delta\left[\left(\psi_{y} \psi_{y x y}-\psi_{x} \psi_{y y y}\right)+\delta^{2}\left(\psi_{y} \psi_{x x x}-\psi_{x} \psi_{x y x}\right)\right] \\
& \quad=\delta^{4} \psi_{x x x x}+2 \delta^{2} \psi_{x x y y}+\psi_{y y y y} .
\end{aligned}
$$

Under the assumptions of long wavelength, $\delta \ll 1$ and low Reynolds number, Equation (8) becomes

$$
\psi_{\text {yyyy }}=0 .
$$

Also, the upper and lower channel walls will become

$$
\begin{aligned}
& y=h_{1}=1+a \cos 2 \pi x, \\
& y=h_{2}=-d-b(\cos 2 \pi x+\phi) .
\end{aligned}
$$

The non-dimensional boundary conditions are

$$
\begin{aligned}
\psi & =\frac{q}{2} \quad \text { at } \quad y=h_{1}, \\
\psi & =-\frac{q}{2} \quad \text { at } \quad y=h_{2}, \\
\frac{\partial \psi}{\partial y}+\beta \frac{\partial^{2} \psi}{\partial y^{2}} & =-1 \quad \text { at } \quad y=h_{1}, \\
\frac{\partial \psi}{\partial y}-\beta \frac{\partial^{2} \psi}{\partial y^{2}} & =-1 \quad \text { at } \quad y=h_{2},
\end{aligned}
$$

where $q$ is the flux between the two walls in the wave frame and $\beta=\eta / d_{1}$ is the non-dimensional slip parameter. The first and second conditions come from the relation 


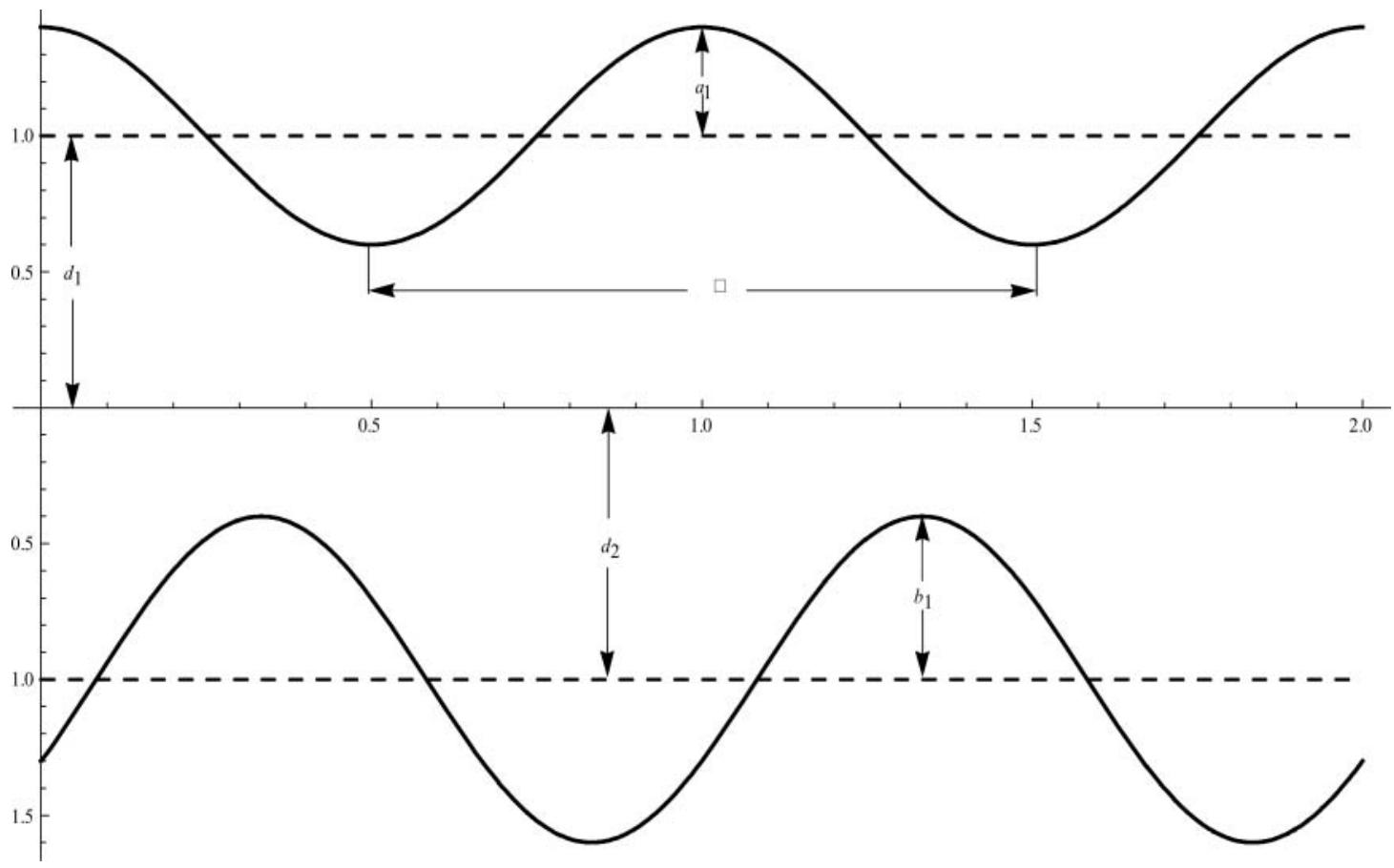

Figure 1. Geometry of the problem.

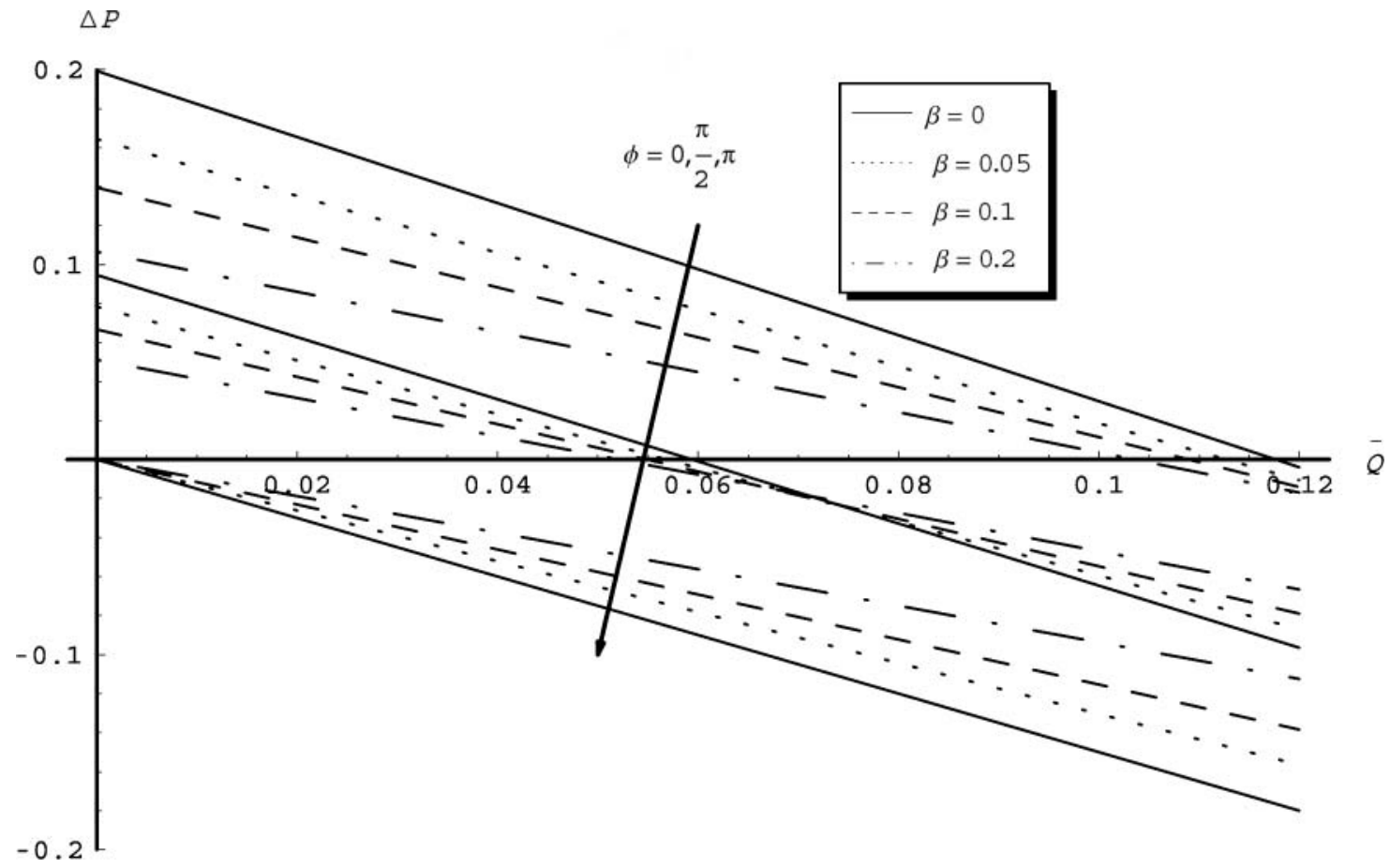

Figure 2. The variation of $\bar{Q}$ with $\Delta p$ for $a=b=0.2$ and $d=1$, for different values of $\phi$ and $\beta$. 


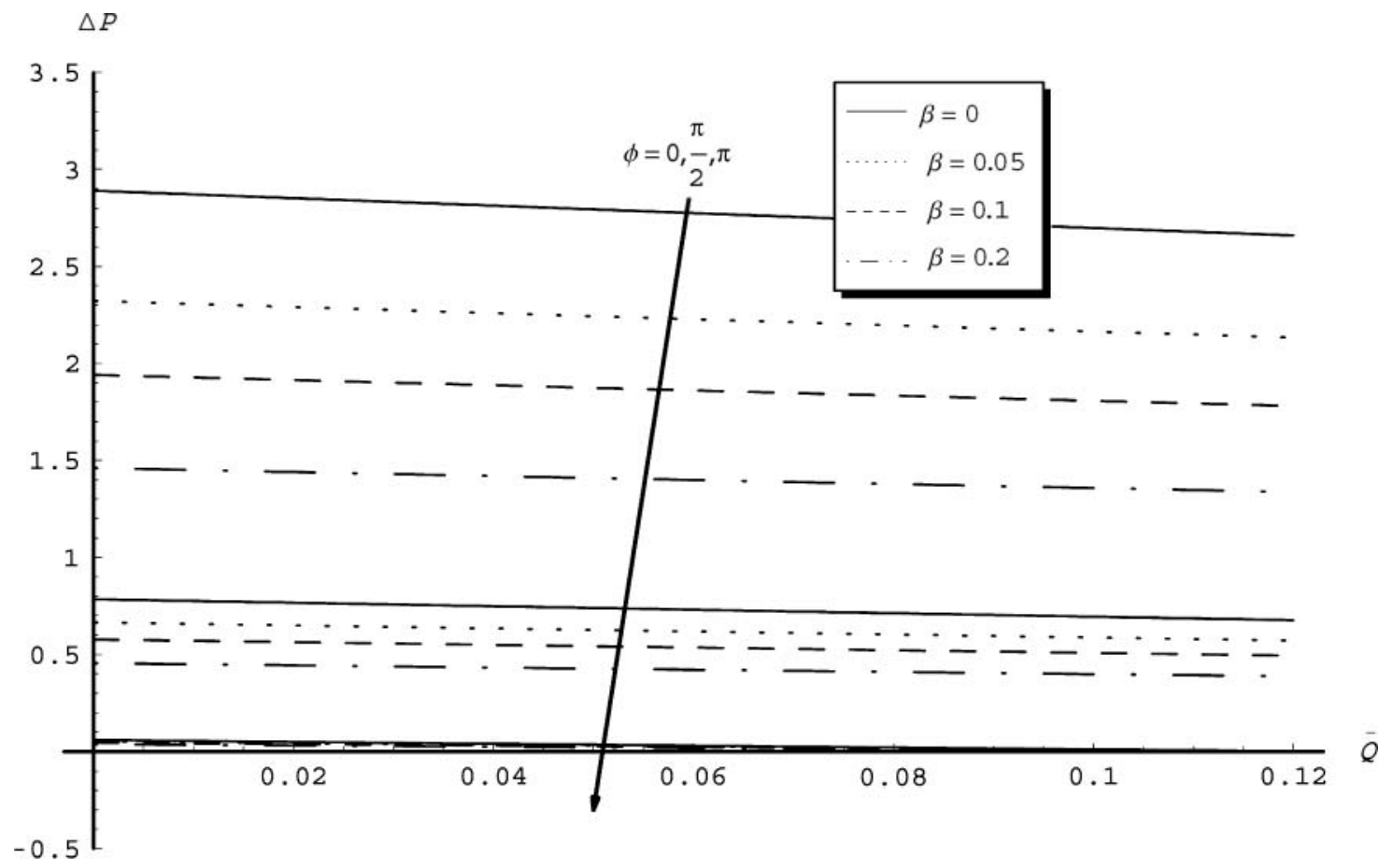

Figure 3. The variation of $\bar{Q}$ with $\Delta p$ for $a=0.7, b=1.2$ and $d=2$, for different of $\phi$ and $\beta$.

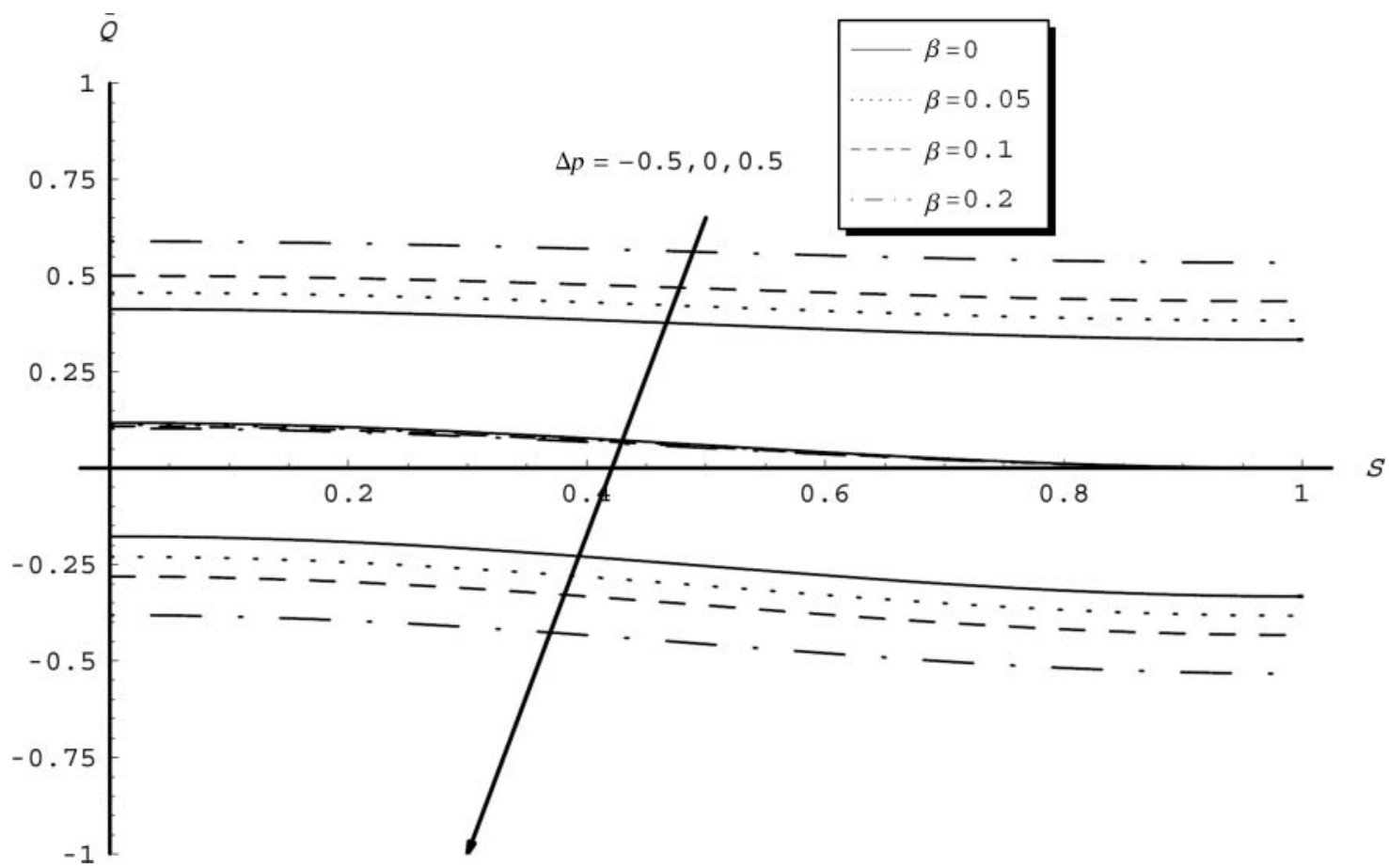

Figure 4. The variation of $\bar{Q}$ with $\phi$ for $a=b=0.5$ and $d=1$, for different values of $\Delta p$ and $\beta$. 
between the flux $q$ and the stream function $\psi$, where $q=$ $\psi\left(h_{1}\right)-\psi\left(h_{2}\right)$. The third and fourth conditions come from the slip boundary condition $(u=\mp \eta(\partial u / \partial y))$.

Using these boundary conditions, the solution of Equation (15) is

$$
\begin{aligned}
\psi= & \left(y-\frac{h_{1}+h_{2}}{2}\right)\left[-1+\left(q+h_{1}-h_{2}\right)\right. \\
& \times\left(\frac{-2\left(y-\frac{h_{1}+h_{2}}{2}\right)^{2}}{\left(h_{1}-h_{2}\right)^{2}\left(6 \beta+h_{1}-h_{2}\right)}\right. \\
& \left.\left.+\frac{3\left(4 \beta+h_{1}-h_{2}\right)}{2\left(h_{1}-h_{2}\right)\left(6 \beta+h_{1}-h_{2}\right)}\right)\right] .
\end{aligned}
$$

In the limit as $\beta \rightarrow 0$ (no slip flow), we get

$$
\begin{aligned}
\psi= & \frac{q+h_{1}-h_{2}}{\left(h_{2}-h_{1}\right)^{3}}\left(2 y^{3}-3\left(h_{1}+h_{2}\right) y^{2}+6 h_{1} h_{2}\right)-y \\
& +\frac{1}{\left(h_{2}-h_{1}\right)^{3}}\left[\left(\frac{q}{2}+h_{1}\right)\left(h_{2}^{3}-3 h_{1} h_{2}^{2}\right)\right. \\
& \left.-\left(h_{2}-\frac{q}{2}\right)\left(h_{1}^{3}-3 h_{2} h_{1}^{2}\right)\right]
\end{aligned}
$$

which is the same as the result obtained by Mishra and Ramachandra Rao (2003).

\section{The average volume flow rate}

The flux at any axial station in the fixed frame is given by

$$
Q=\int_{H_{1}}^{H_{2}} U(y) d Y
$$

Integrating the non-dimensional form of this equation we have

$$
Q=q+h_{1}-h_{2}
$$

The average volume flow rate over one period, $T=\lambda / c$, of the peristaltic wave is defined as

$$
\bar{Q}=\frac{1}{T} \int_{0}^{T} Q d T
$$

By using Equation (19) in Equation (20) we get

$$
\bar{Q}=\frac{1}{T} \int_{0}^{T}\left(q+h_{1}-h_{2}\right) d T=q+d+1
$$

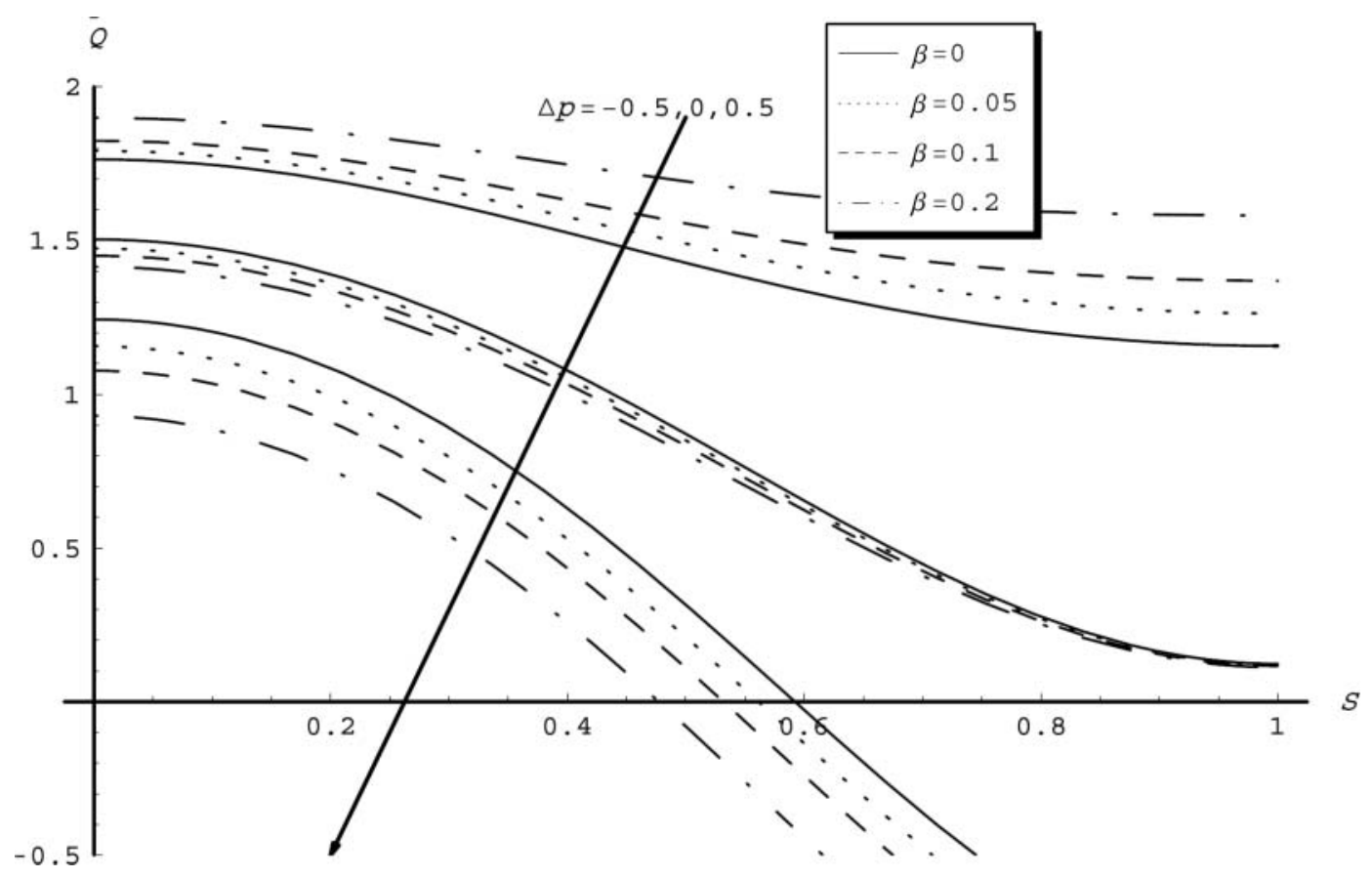

Figure 5. The variation of $\bar{Q}$ with $\phi$ for $a=0.7 b=1.2$ and $d=2$, for different values of $\Delta p$ and $\beta$. 


\section{The pressure gradient}

The pressure gradient obtained from the dimensionless momentum equation for the axial velocity is as follows:

$$
\frac{d p}{d x}=\frac{\partial^{3} \psi}{\partial y^{3}}
$$

From Equation (16) we get

$$
\frac{d p}{d x}=\frac{-12\left(q+h_{1}-h_{2}\right)}{\left(6 \beta+h_{1}-h_{2}\right)\left(h_{1}-h_{2}\right)^{2}}
$$

through substitution from Equation (21) into Equation (23). Then

$$
\frac{d p}{d x}=\frac{-12\left(\bar{Q}-(1+d)+h_{1}-h_{2}\right)}{\left(6 \beta+h_{1}-h_{2}\right)\left(h_{1}-h_{2}\right)^{2}} .
$$

In the limit as $\beta \rightarrow 0$ (no slip flow), we get

$$
\frac{d p}{d x}=-12\left[\frac{1}{\left(h_{1}-h_{2}\right)^{2}}+\frac{(\bar{Q}-(1+d))}{\left(h_{1}-h_{2}\right)^{3}}\right]
$$

which is the same as the result obtained by Mishra and Ramachandra Rao (2003).

\section{Discussion of the results}

\subsection{Pumping characteristics}

By integrating the axial pressure gradient (24) over one wavelength $\lambda$, the pressure rise is given by

$$
\begin{aligned}
\Delta p & =\int_{0}^{1} \frac{d p}{d x} d x \\
& =\int_{0}^{1} \frac{-12\left(\bar{Q}-(1+d)+h_{1}-h_{2}\right)}{\left(6 \beta+h_{1}-h_{2}\right)\left(h_{1}-h_{2}\right)^{2}} d x,
\end{aligned}
$$

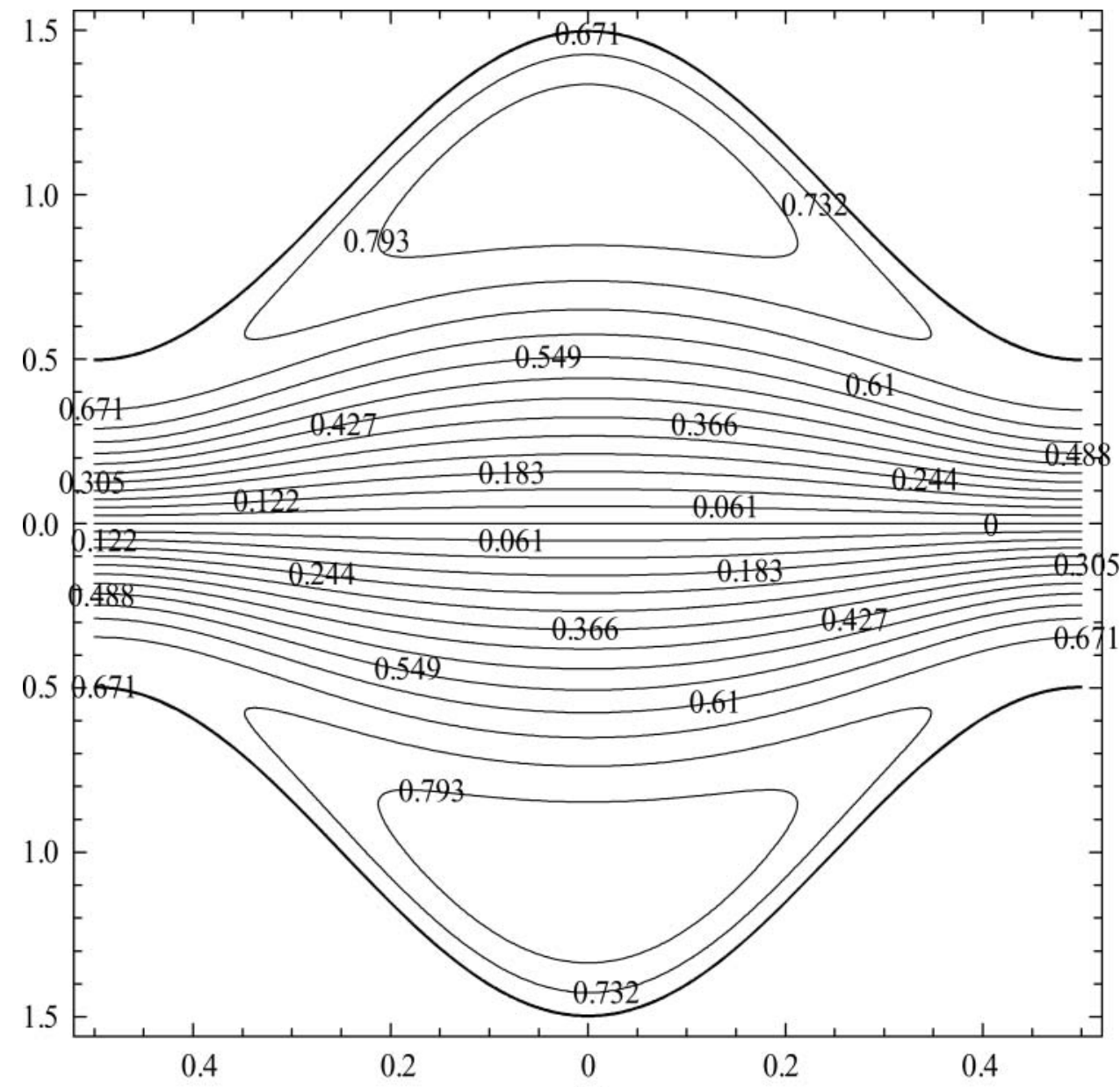

(a)

Figure 6. Streamlines for $a=b=0.5, d=1, \phi=0$ and $\bar{Q}=2 \bar{Q}_{+}$for different values of $\beta$ : (a) $\beta=0$, (b) $\beta=0.08$, (c) $\beta=0.15$ and (d) $\beta=0.2$. 


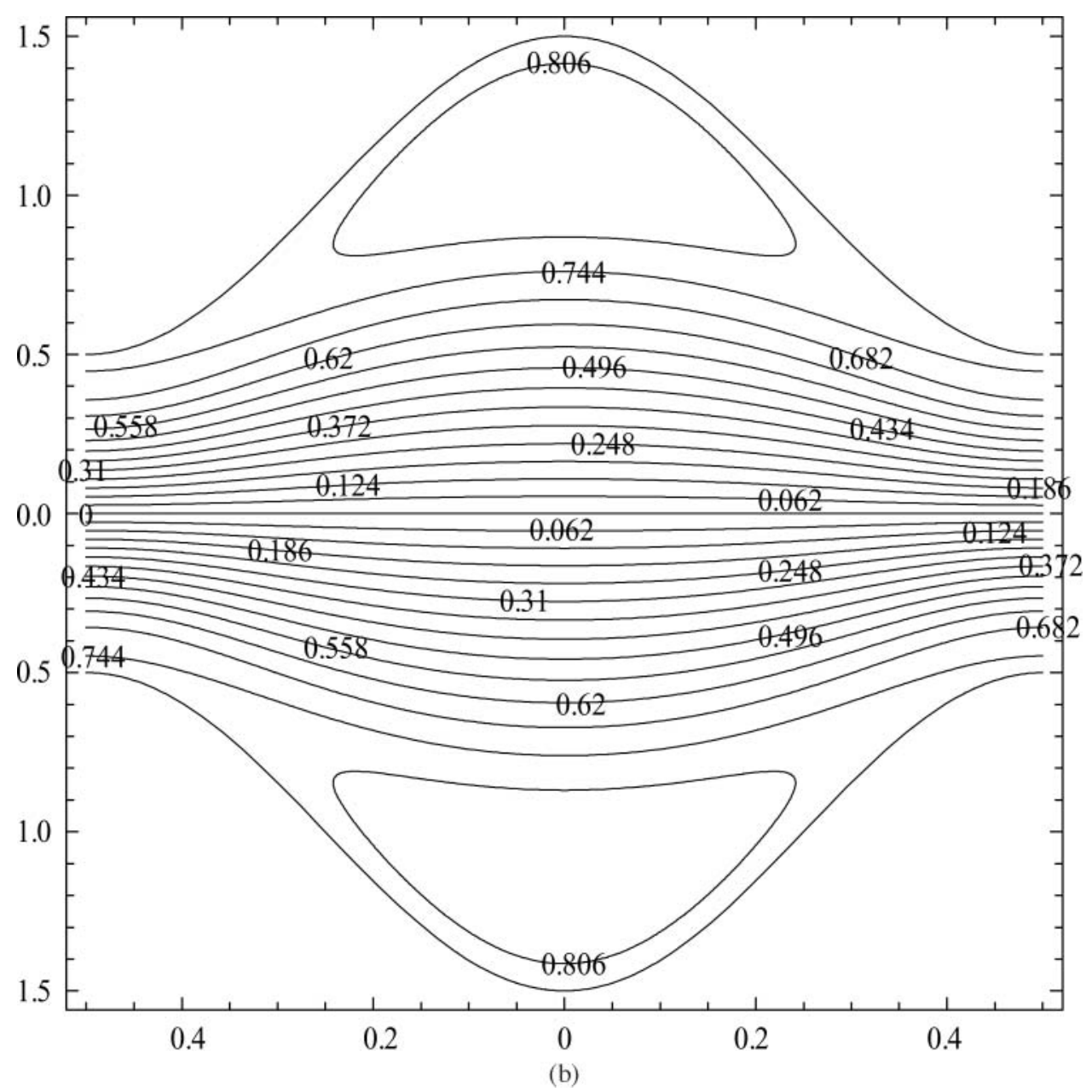

Figure 6. (Continued)

which can be written as

$$
\Delta p=-12\left((\bar{Q}-(1+d)) I_{1}+I_{2}\right),
$$

where

$$
I_{1}=\int_{0}^{1} \frac{1}{\left(6 \beta+h_{1}-h_{2}\right)\left(h_{1}-h_{2}\right)^{2}} d x
$$

and

$$
I_{2}=\int_{0}^{1} \frac{1}{\left(6 \beta+h_{1}-h_{2}\right)\left(h_{1}-h_{2}\right)} d x
$$

Also, we can write the average volume flow rate in terms of the pressure rise as follows:

$$
\bar{Q}=(1+d)-\frac{\frac{\Delta p}{12}+I_{2}}{I_{1}} .
$$

The maximum pressure rise $\Delta p_{0}$ is obtained by putting $\bar{Q}=0$, i.e.

$$
\Delta p_{0}=12\left((1+d) I_{1}-I_{2}\right) .
$$

The maximum volume flow rate $\bar{Q}_{0}$ is obtained by putting $\Delta p=0$, i.e.

$$
\bar{Q}_{0}=(1+d)-\frac{I_{2}}{I_{1}} .
$$

In the limit as $\beta \rightarrow 0$ (no slip flow),

$$
\begin{aligned}
\Delta p & =\int_{0}^{1} \frac{d p}{d x} d x \\
& =-12 \int_{0}^{1}\left[\frac{1}{\left(h_{1}-h_{2}\right)^{2}}+\frac{(\bar{Q}-(1+d))}{\left(h_{1}-h_{2}\right)^{3}}\right] d x,
\end{aligned}
$$

which is the as the same result obtained by Mishra and Ramachandra Rao (2003). 


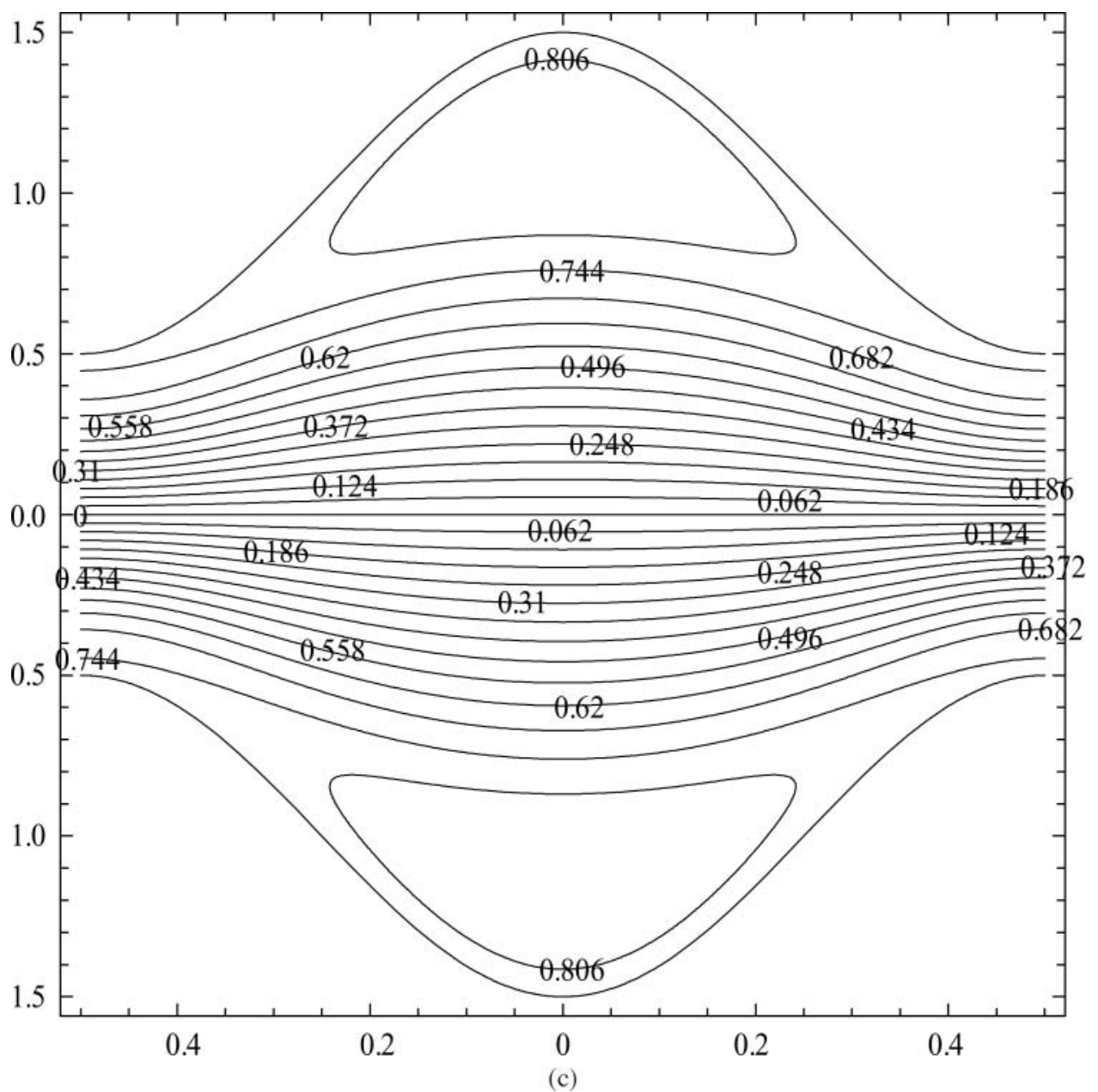

Figure 6. (Continued)

Figure 2 represents the variation of volume flow rate $\bar{Q}$ with the pressure rise $\Delta p$, for different values of the phase difference $\phi$, when the peristaltic wave amplitudes are the same $(a=b=0.2, d=1)$, taking into account different values of the dimensionless slip parameter $\beta$ ( $\beta=$ $0,0.05,0.1,0.2)$. When $\phi=0$, it is observed that $\Delta p$ has maximum value when $\bar{Q}=0\left(\Delta p=\Delta p_{0}\right.$ for $\left.\bar{Q}=0\right)$, and the pressure rise $\Delta p$ decreases with increase in $\bar{Q}$ until $\Delta p$ becomes zero for the maximum value of $\bar{Q}\left(\bar{Q}=\bar{Q}_{0}\right.$ for $\Delta p=0)$. When $\phi=\pi / 2, \Delta p$ has values smaller than it has when $\phi=0 ; \Delta p$ decreases with increase in $\beta$ and $\bar{Q}$, but after a critical value of $\bar{Q}$ we observe that $\Delta p$ decreases with $\beta$ but still decreases with increase in $\bar{Q}$. Also, this observation remains for $\phi=\pi$, where $\Delta p$ begins from a negative value.

Figure 3 displays the variation of $\Delta p$ with the volume flow rate $\bar{Q}$ for different values of $\phi$ and $\beta$ in the case of different peristaltic wave amplitudes $(a=0.7, b=1.2, d=$
2). From Figures 2 and 3 we can see that $\Delta p$ decreases slowly with $\bar{Q}$ for waves of different amplitudes than for waves of equal amplitudes.

The variation of volume flow rate $\bar{Q}$ with the phase difference $\phi(\phi=\pi S, 0 \leq S \leq 1)$, for different values of the pressure rise $\Delta p(\Delta p=-0.5,0,0.5)$ and the slip parameter $\beta(\beta=0,0.05,0.1,0.2)$ at the peristaltic wave amplitudes $(a=b=0.5, d=1)$, is represented in Figure 4. We observe from this figure that when $\Delta p=-0.5, \bar{Q}$ increases as $\beta$ increases and remains positive for all values of $S$. When $\Delta p=0$, we observe that $\bar{Q}$ decreases and approaches zero as $S \rightarrow 1(\phi \rightarrow \pi)$ for all values of $\beta$. When $\Delta p=0.5$, we observe that $\bar{Q}$ has negative values for all values of $S$ and decreases as $\beta$ increases.

Figure 5 is the same as Figure 3 but with different peristaltic wave amplitudes $(a=0.7, b=1.2, d=2)$, and we can see that the relation between $\bar{Q}$ and $S$ is a non-linear function and rapidly decreases with $S$. 


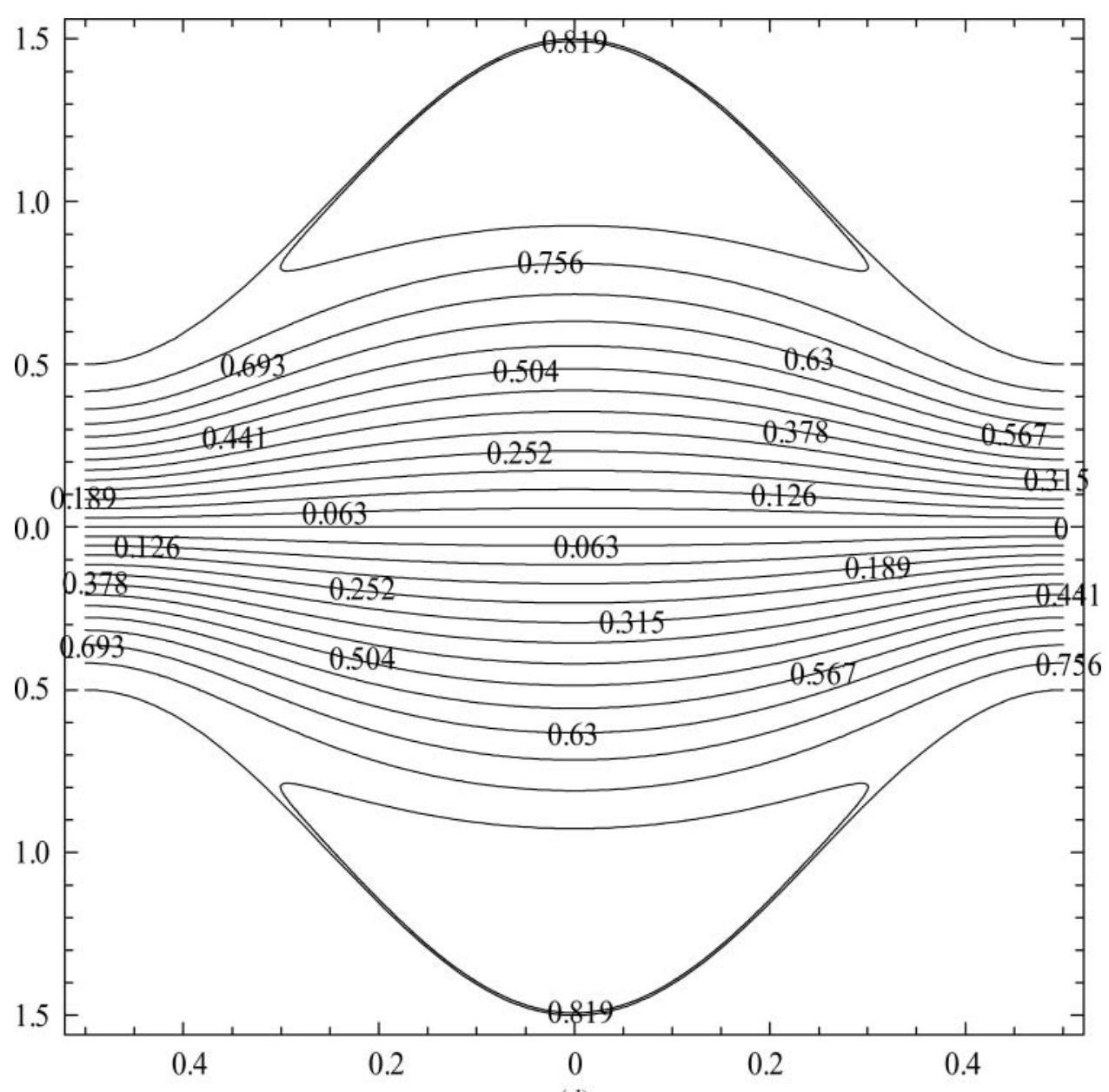

(d)

Figure 6. (Continued)

\section{2. $\quad$ Trapping}

The phenomenon of trapping is the manner, whereby a bolus (defined as the volume of a fluid bounded by closed streamlines in the wave frame) is transported at a wave speed as if trapped by the wave (Eytan et al. 2001; Gharsseldien 2003; Mishra and Rao 2003; El-Hakeem et al. 2006). The trapping limits are determined by calculating the ratio between the minimum volume flow rate $Q_{-}$and the maximum volume flow rate $Q_{+}$, where $\psi=0$ and $y \neq 0$ (El-Hakeem et al. 2006). So by equating (16) to zero and solving it with respect to $y$ we get that $y_{1}=\left(h_{1}+h_{2}\right) / 2$ is one of the three roots of this equation. So, we can write

$$
\begin{aligned}
& -\frac{1}{\left(q+h_{1}-h_{2}\right)}\left[2 y^{2}\left(-q-h_{1}+h_{2}\right)+2 y\left(h_{1}^{2}-h_{2}^{2}\right.\right. \\
& \left.\quad+q h_{1}+q h_{2}\right)+q\left(h_{1}^{2}+h_{2}^{2}-4 h_{1} h_{2}+6 \beta\left(h_{1}-h_{2}\right)\right) \\
& \left.\quad-2 h_{1}^{2} h_{2}+2 h_{1} h_{2}^{2}\right]=0 .
\end{aligned}
$$

This equation gives two real roots for $y$, when the discriminate

$$
\frac{q+\frac{\left(h_{1}-h_{2}\right)^{2}}{3\left(4 \beta+h_{1}-h_{2}\right)}}{q+h_{1}-h_{2}} \geq 0 .
$$

By simplifying this equation we get

$$
q \rightarrow-\frac{\left(h_{1}-h_{2}\right)^{2}}{3\left(4 \beta+h_{1}-h_{2}\right)}
$$

So we can say that Equation (30) gives two real roots if $\bar{Q}$ satisfies the condition

$$
(1+d)-\frac{F_{\min }^{2}}{3\left(4 \beta+F_{\min }\right)}>\bar{Q}>(1+d)-\frac{F_{\max }^{2}}{3\left(4 \beta+F_{\max }\right)},
$$


where $F=h_{1}-h_{2}=1+d+a \cos (2 \pi x)+b \cos (2 \pi x+$ $\phi)$, which leads to

$$
\begin{aligned}
& F_{\max }=1+d+\sqrt{a^{2}+b^{2}+2 a b \cos \phi}, \\
& F_{\min }=1+d-\sqrt{a^{2}+b^{2}+2 a b \cos \phi} .
\end{aligned}
$$

To determine the ratio between the minimum volume flow rate and the maximum volume flow rate, we will choose

$$
\bar{Q}_{+}=(1+d)-\frac{F_{\min }^{2}}{3\left(4 \beta+F_{\min }\right)}
$$

and

$$
\bar{Q}_{-}=(1+d)-\frac{F_{\max }^{2}}{3\left(4 \beta+F_{\max }\right)} .
$$

In the limit as $\beta \rightarrow 0$ (no slip flow), we get

$$
\begin{array}{r}
\frac{2(1+d)-\sqrt{a^{2}+b^{2}+2 a b \cos \phi}}{3}<\bar{Q} \\
<\frac{2(1+d)+\sqrt{a^{2}+b^{2}+2 a b \cos \phi}}{3},
\end{array}
$$

which is the same as the result obtained by Mishra and Ramachandra Rao (2003).

By using the relation between $\bar{Q}, \bar{Q}_{+}$and $\bar{Q}_{-}$in the current study, we can explain that the trapping occurs at two regions of the channel, one at the channel centre (central trapping) and the other at the walls (peripheral trapping). The central trapping is observed when $\bar{Q}_{-}<\bar{Q}<\bar{Q}_{+}$; the trapping moves far from the centre line towards the walls when $\bar{Q}>\bar{Q}_{+}$.

In the case of the central trapping $\left(\bar{Q}_{-}<\bar{Q}<\bar{Q}_{+}\right)$, it is observed that the effect of the slip is very weak; so we can say that there is no effect of the slip on the trapping phenomenon in the centre of the channel.

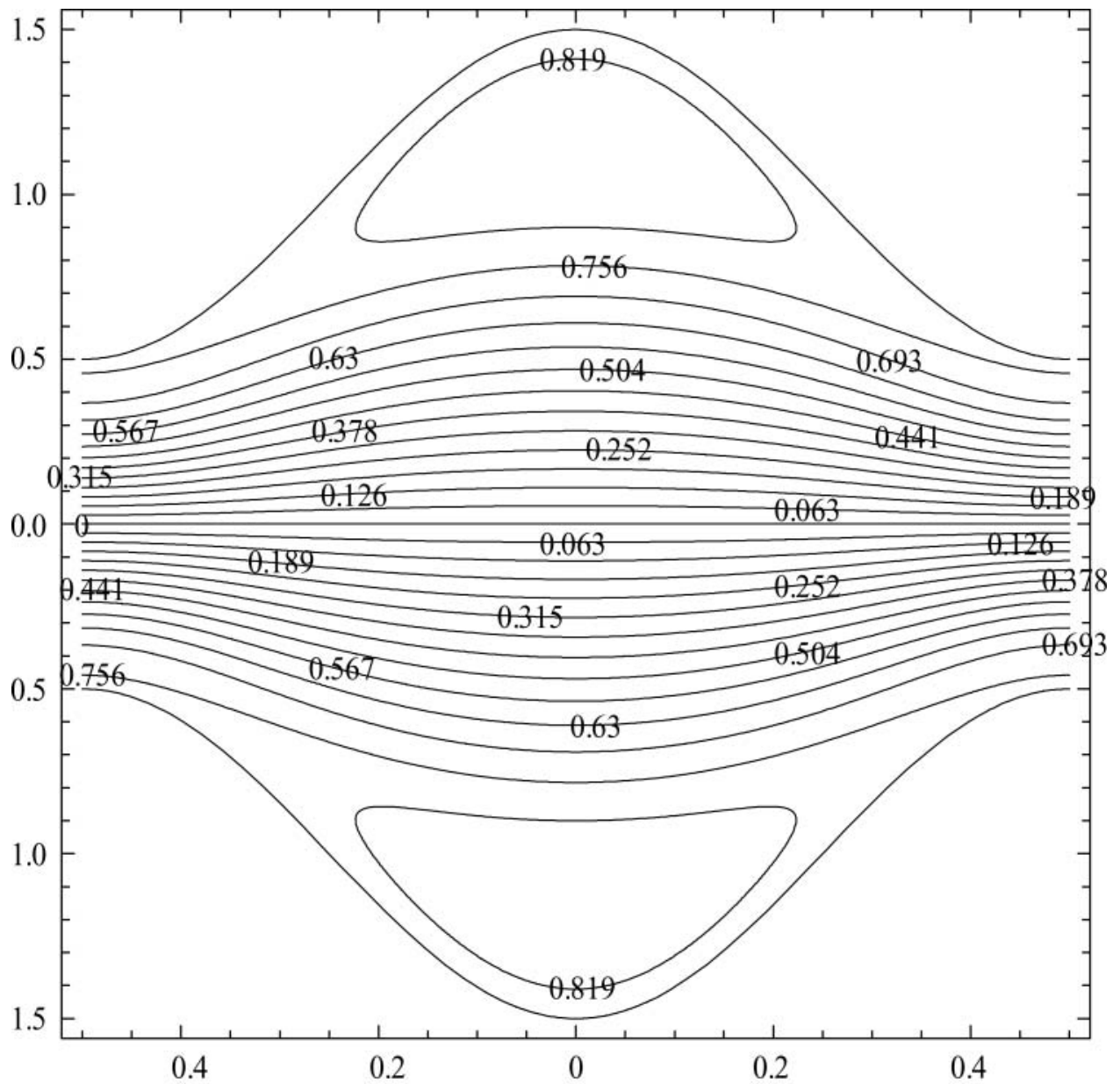

(a)

Figure 7. Streamlines for $a=b=0.5, d=1, \beta=0.1$ and $\bar{Q}=2 \bar{Q}_{+}$for different values of $\phi$ : (a) $\phi=0$, (b) $\phi=\frac{\pi}{2}$ and (c) $\phi=\pi$. 


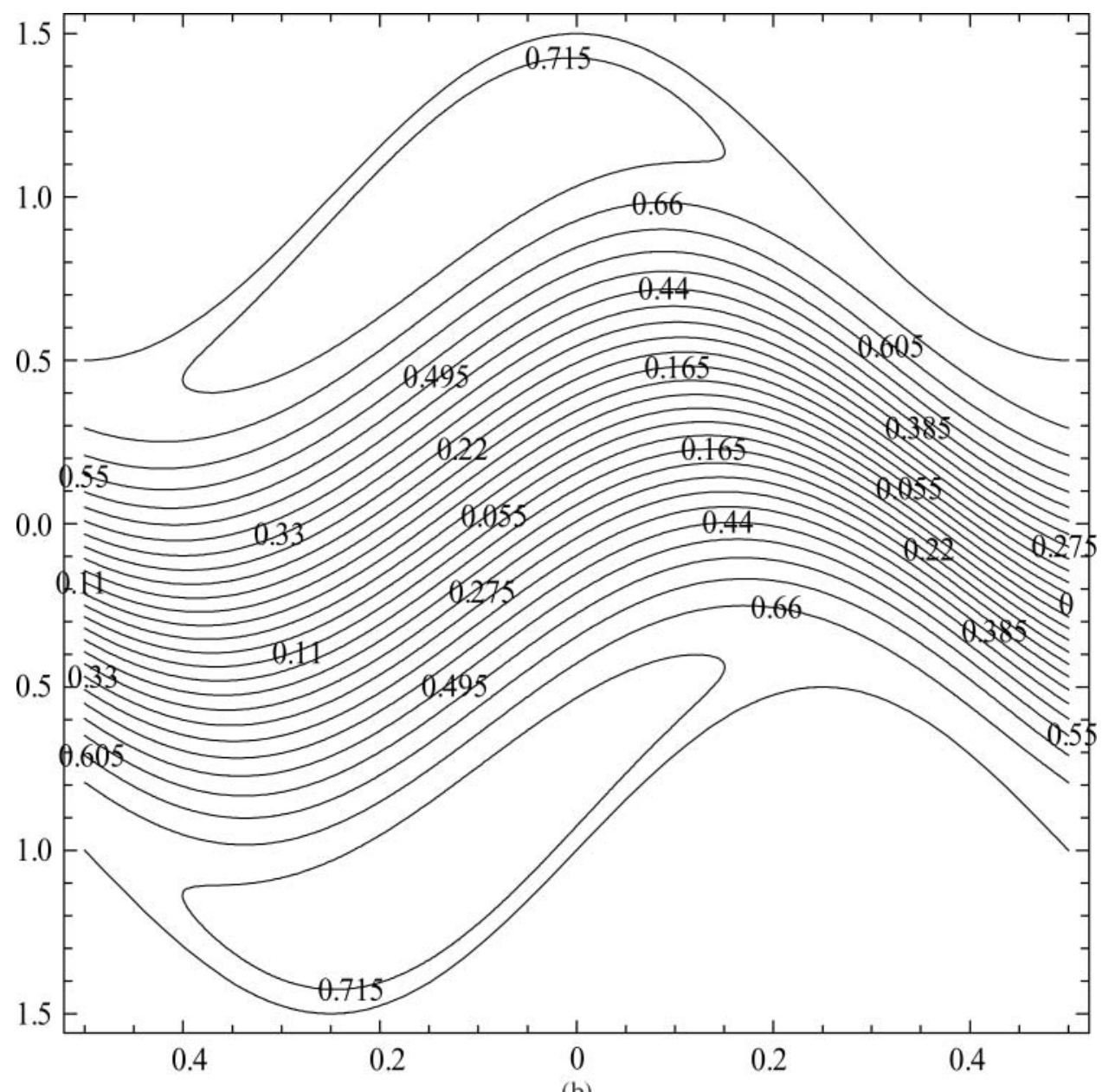

(b)

Figure 7. (Continued)

Figure 6 explains the streamlines when $\bar{Q}>\bar{Q}_{+}$for $\beta=0,0.08,0.15,0.2$ and the wave amplitudes $a=b=$ 0.5 and $d=1$; it is observed that the bolus of the trapping will travel to the walls (peripheral trapping), and furthermore, the size of the trapping bolus increases and the centre of the trapping moves closer to the walls as $\beta$ increases.

Figure 7 investigates the effect of the phase shift $\phi$ on the trapping when $\bar{Q}=2 \bar{Q}_{+}$. In Figure 7, the wave amplitudes are $a=b=0.5$ and $d=1$ and the slip parameter $\beta=0.1$, and $\phi$ takes the values $0, \pi / 2$ and $\pi$. It is observed that the bolus of the trapping will travel to the walls (peripheral trapping), and further, the size of the trapping bolus increases and the centre of trapping moves closer to the walls as $\phi$ increases.

\subsection{Reflux}

Reflux is defined as the presence of some fluid particles whose mean motion over one cycle is against the net pumping direction. Following Shapiro et al. (1969), $Q_{\psi}$ is defined as the dimensionless volume flow rate in the laboratory frame between the centre line of the channel $\left(\left(h_{1}+h_{2}\right) / 2\right)$ and the wave frame streamline $\psi$, which is an indicator of material particles in a fixed frame, and is given by

$$
Q_{\psi}=\int_{\frac{h_{1}+h_{2}}{2}}^{Y(\psi, X, t)} U(X, Y, t) d Y
$$

After using the transformations (6) and the nondimensional variables (7), we get

$$
\begin{aligned}
Q_{\psi}= & \int_{\frac{h_{1}+h_{2}}{2}}^{y(\psi, x, t)} u d y+\int_{\frac{h_{1}+h_{2}}{2}}^{y(\psi, x, t)} d y=\psi+y(\psi, x, t) \\
& -\frac{h_{1}+h_{2}}{2}
\end{aligned}
$$




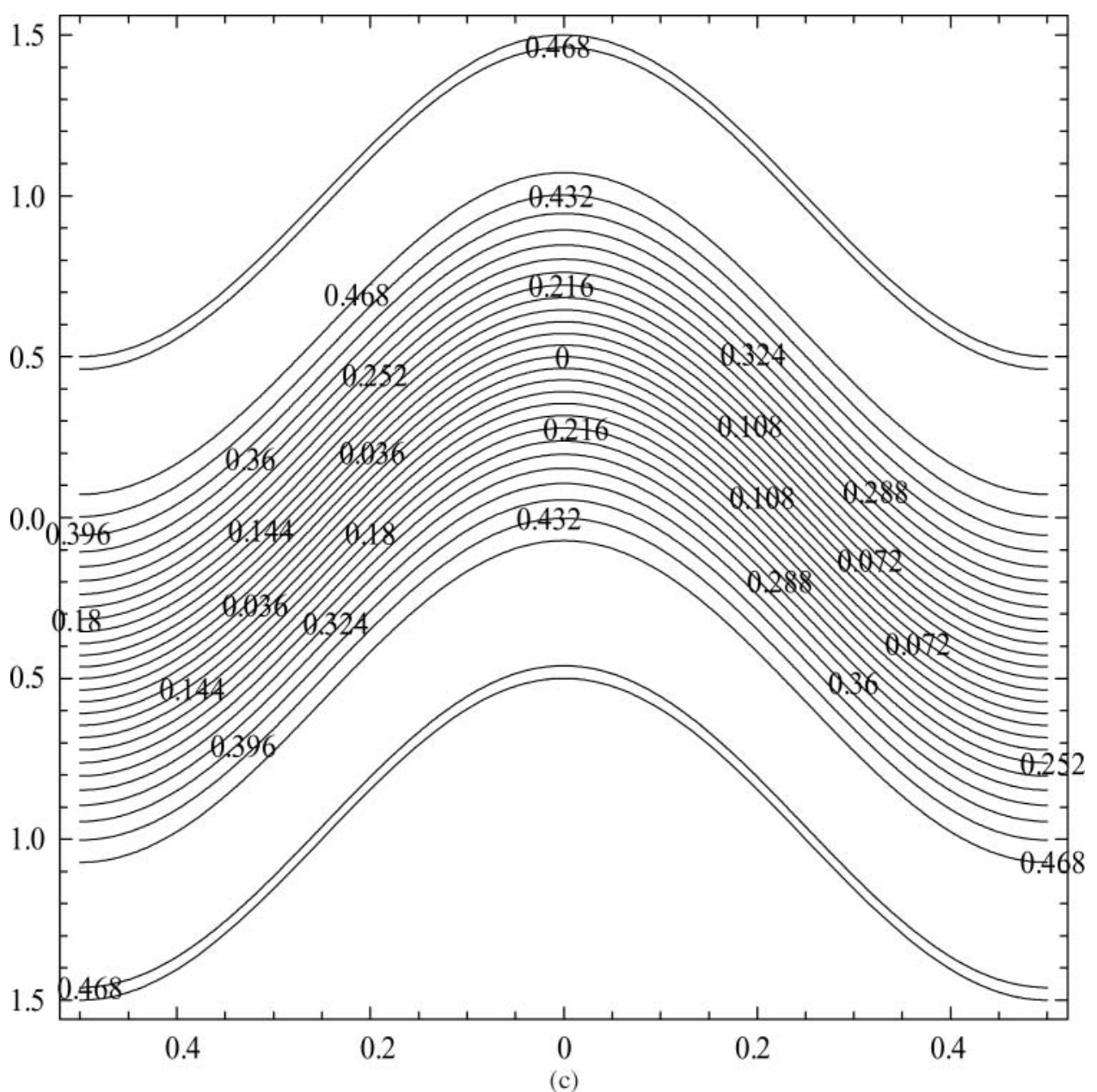

Figure 7. (Continued)

Averaging this equation over one period of the wave, we get

$$
Q_{\psi}=\psi+\int_{0}^{1} y(\psi, x) d x-\frac{1-d}{2} .
$$

A reflux layer exists near the wall whenever $Q^{*}>1$, where $Q^{*}=Q_{\psi} / Q_{w}=2 Q_{\psi} / \bar{Q}$.

In order to calculate the limits on $\bar{Q}$ for reflux, we will consider a wave streamline very close to the wall; so we will expand $y$ about the wall in powers of a small parameter $\epsilon$, where

$$
\epsilon=\psi-\psi_{w}=\psi-\frac{q}{2} .
$$

Then

$$
\psi=\epsilon+\frac{q}{2},
$$

and we will take

$$
y=h_{1}+a \epsilon+b \epsilon^{2}+\ldots .
$$

Substituting Equation (16) into Equation (39) and then using Equation (40), we can calculate after some simplifications the values of coefficients $a, b, \ldots$ Then $y$ takes the form

$$
\begin{aligned}
y= & h_{1}-\epsilon+\frac{6 \beta\left(q+h_{1}-h_{2}\right) \epsilon}{6 q \beta-\left(h_{1}-h_{2}\right)^{2}} \\
& -\frac{3\left(q+h_{1}-h_{2}\right)\left(h_{1}-h_{2}\right)^{2}\left(6 \beta+h_{1}-h_{2}\right)^{2} \epsilon^{2}}{\left(-6 q \beta+\left(h_{1}-h_{2}\right)^{2}\right)^{3}} \\
& +\ldots
\end{aligned}
$$




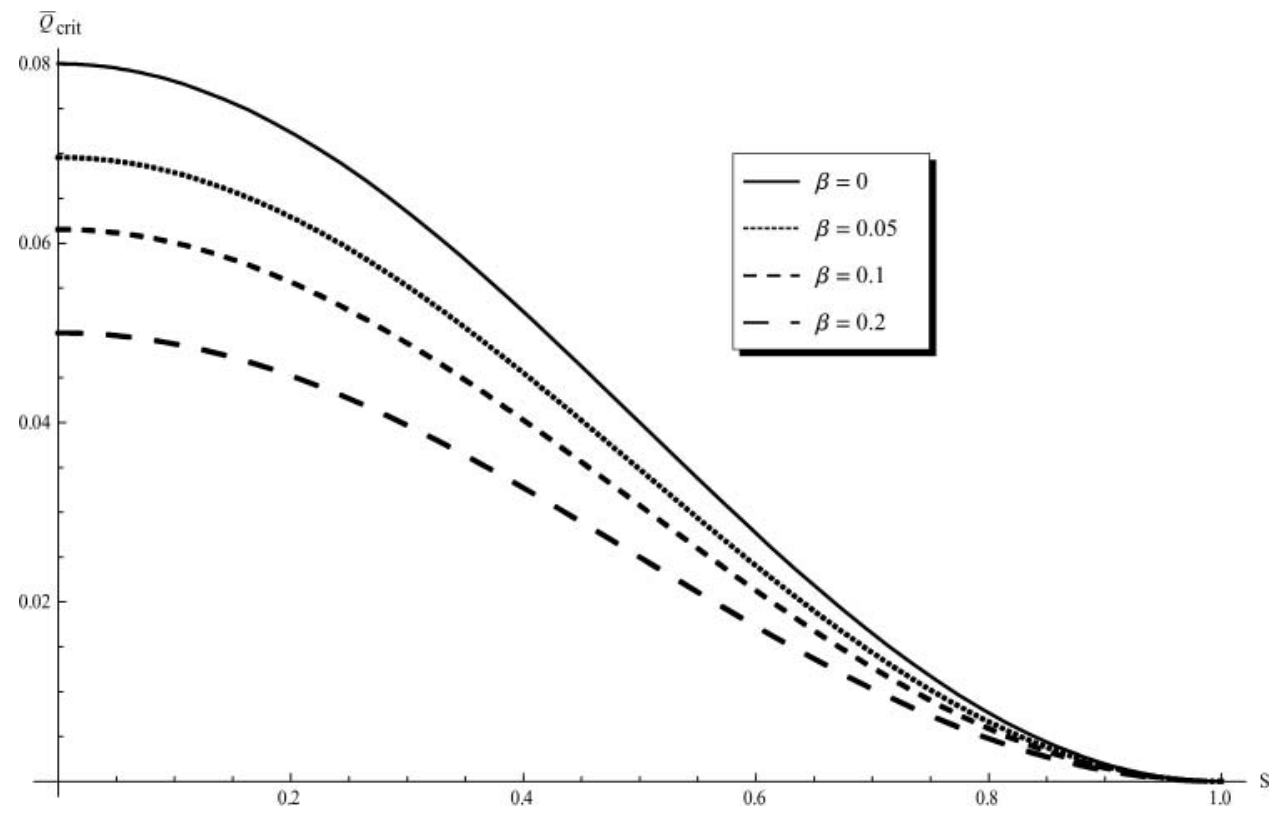

Figure 8. Reflux limits with the phase difference for $a=b=0.2$ and $d=1$, or different values of $\beta$.

Integrating $y$ with respect to $x$ and neglecting that term which contains $\epsilon^{2}$, we have

$$
\begin{aligned}
& \int_{0}^{1} y(\psi, x) d x=1-\epsilon+\epsilon \\
& \times\left[\frac{\sqrt{\frac{3 \beta}{2}}(\sqrt{q}-\sqrt{6 \beta})}{\left((1+d+\sqrt{6 q \beta})^{2}-\left(a^{2}+b^{2}+2 a b \cos \phi\right)\right)^{\frac{1}{2}}}\right. \\
& \left.-\frac{\sqrt{\frac{3 \beta}{2}}(\sqrt{q}+\sqrt{6 \beta})}{\left((1+d-\sqrt{6 q \beta})^{2}-\left(a^{2}+b^{2}+2 a b \cos \phi\right)\right)^{\frac{1}{2}}}\right] .
\end{aligned}
$$

Substituting from Equations (38) and (41) into Equation (37), we obtain

$$
\begin{aligned}
Q_{\psi} & =\frac{\bar{Q}}{2}+\epsilon \\
& \times\left[\frac{\sqrt{\frac{3 \beta}{2}}(\sqrt{q}-\sqrt{6 \beta})}{\left((1+d+\sqrt{6 q \beta})^{2}-\left(a^{2}+b^{2}+2 a b \cos \phi\right)\right)^{\frac{1}{2}}}\right. \\
& \left.-\frac{\sqrt{\frac{3 \beta}{2}}(\sqrt{q}+\sqrt{6 \beta})}{\left((1+d-\sqrt{6 q \beta})^{2}-\left(a^{2}+b^{2}+2 a b \cos \phi\right)\right)^{\frac{1}{2}}}\right] .
\end{aligned}
$$

Applying the reflux condition $Q^{*}>1$ which we refer to, we get that the reflux limits are given when

$$
\bar{Q}<\bar{Q}_{\text {crit }},
$$

where

$$
\bar{Q}_{\text {crit }}=\frac{a^{2}+b^{2}+2 a b \cos \phi}{1+d+6 \beta} .
$$

At this value the of $Q_{\text {crit }}$ reflux limits are observed.

Finally, our results about reflux agree with those of Mishra and Ramachandera (2003) when $\beta \rightarrow 0$ (no slip flow) for every equation.

Figure 8 investigates the influence of the slip parameter $\beta$ and the phase difference $\phi=\pi S(0 \leq S \leq 1)$ on the reflux limits, when the peristaltic wave amplitudes are $a=$ $b=0.2$ and $d=1$. We observe that the reflux limits have the maximum value (0.08) when $\beta=0$ and $\phi=0$ (i.e. $S=$ 0 ; symmetric channel). Also, the reflux limits approaches zero when the phase difference $\phi \rightarrow \pi$ or $S \rightarrow 1$, which means that the channel becomes asymmetric, for any value of $\beta$.

Finally we observe that the values of the reflux limits decrease with increase in $\beta$ and $\phi$. So we can say that there is an obvious influence of the slip parameter and the phase difference on the value of $\bar{Q}_{\text {crit }}$ at which the reflux limits occur.

\section{References}

Abd El-Hakeem Abd El-Naby, El-Misery, AEM, Abd El-Kareem, MF. 2006. Effects of a magnetic field on trapping through peristaltic motion for generalized Newtonian fluid in channel. Physica A 367:79-92.

Ali N, Hayat T. 2007. Peristaltic motion of a Carreau fluid in an asymmetric channel. Appl. Math. Comput. 193(2):535-552. 
Ali N, Hayat T. 2008. Peristaltic flow of a micropolar fluid in an asymmetric channel. Comp. Math. Appl. 55(4):589-608.

Ali N, Hussain Q, Hayat T, Asghar S. 2008. Slip effects on the peristaltic transport of MHD fluid with variable viscosity. Phys. Lett. A 372(9):1477-1489.

Ebaid A. 2008. Effects of magnetic field and wall slip conditions on the peristaltic transport of a Newtonian fluid in an asymmetric channel. Phys. Lett. A 372(24):4493-4499.

Ellahi R. 2009. Effects of the slip boundary condition on nonNewtonian flows in a channel. Commun. Nonlin. Sci. Numer. Sim. 14(4):1377-1384.

El-Shehawey EF, El-Dabe NT, El-ghzey EM, Ebaid A. 2006. Peristaltic transport in an asymmetric channel through a porous medium. Appl. Math. Comput. 182:140-150.

El-Shehawey EF, El-Dabe NT, Islam El-Desoky M. 2006. Slip effects on the peristaltic flow of a non-Newtonian Maxwellian fluid. Acta Mech. 186:141-159.

El-Shehawey EF, Husseny SZA. 2000. Effects of porous boundaries on peristaltic transport through a porous medium. Acta Mech. 143:165-177.

Eytan O, Jaffa AJ, Elad D. 2001 Peristaltic flow in a tapered channel: application to embryo transport within the uterine cavity. Med. Eng. Phys. 23:473-482.

Faltas MS, Saad EI. 2005. Stokes flow with slip caused by the axisymmetric motion of a sphere bisected by a free surface bounding a semi-infinite micropolar fluid. Int. J. Eng. Sci. 43:953-976.

Fung YC, Yih CS. 1968. Peristaltic transport. J. Appl. Mech. 35:669-675.

Gharsseldien ZM. 2003. On some problems in biofluidmechanics [PhD dissertation]. [Cairo (Egypt)]: Al-Azhar University.

Hayat T, Hussain Q, Ali N. 2008a. Influence of partial slip on the peristaltic flow in a porous medium. Physica A 387(14):3399_ 3409.
Hayat T, Umar Qureshi M, Ali N. 2008b. The influence of slip on the peristaltic motion of a third order fluid in an asymmetric channel. Phys. Lett. A 372(15):2653-2664.

Joshi YM, Denn MM. 2003. Planar contraction flow with a slip boundary condition. J. Non-Newton. Fluid Mech. 114:185195.

Mekheimer KhS. 2003. Nonlinear peristaltic transport through a porous medium in an inclined planar channel. J. Porous Media 6(3):189-201.

Mishra M, Rao AR. 2003. Peristaltic transport of a Newtonian fluid in an asymmetric channel. Z. Angew. Math. Phys. 54:532550 .

Nakai A, Togashi K, Kosoka K. 2004. Uterine peristalsis: comparison of transvaginal ultrasound and two different sequence of cine MR imaging. J. Magn. Reson. Imaging 20:463469

Nakai A, Togashi K, Yamaoka T. 2003. Uterine peristaltic show on cine MR imaging using ultrafast sequence. J. Magn. Reson. Imaging 18:726-733.

Nishino M, Togashi K, Nakai A. 2005. Uterine contraction evaluated on cine MR imaging in patients with uterine leiomyomas. Eur. J. Radiol. 53(1):142-146.

Rao IJ, Rajagopal KR. 1999. The effect of the slip boundary condition on the flow of fluids in a channel. Acta Mech. 135:113126.

Shapiro AH, Jaffrin MY, Weinberg, SL. 1969. Peristaltic pumping with long wavelengths at low Reynolds number. J. Fluid Mech. 37:799-826.

Srivastava LM, Srivastava VP. 1982. Peristaltic transport of a twolayered model of physiological fluid, J. Biomech. 15(4):257265.

Strandell A, Lindhard A. 2002. Why does hydrosalpinx reduce fertility? The importance of hydrosalpinx fluid. Hum. Reprod. 17(5):1141-1145. 

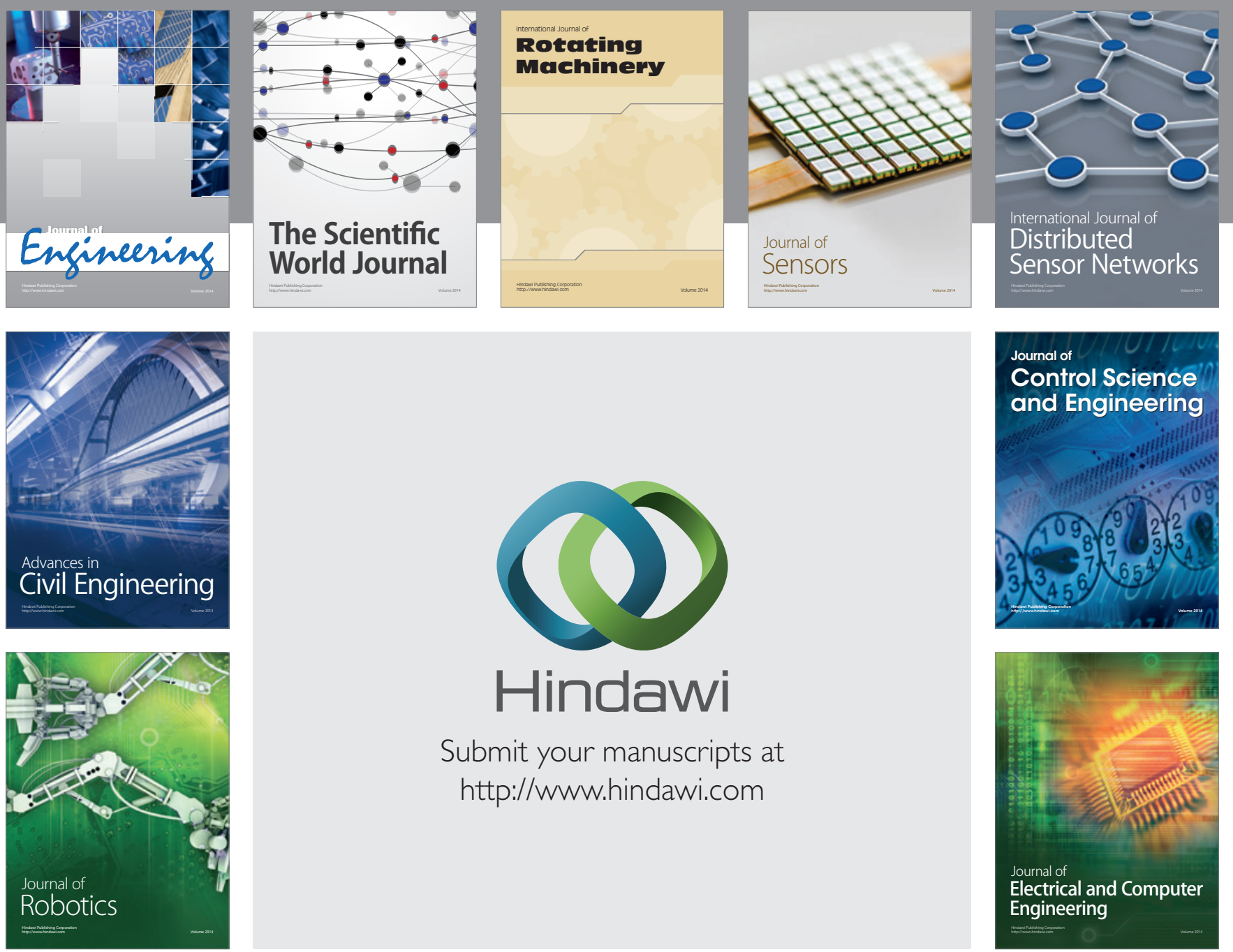

Submit your manuscripts at

http://www.hindawi.com
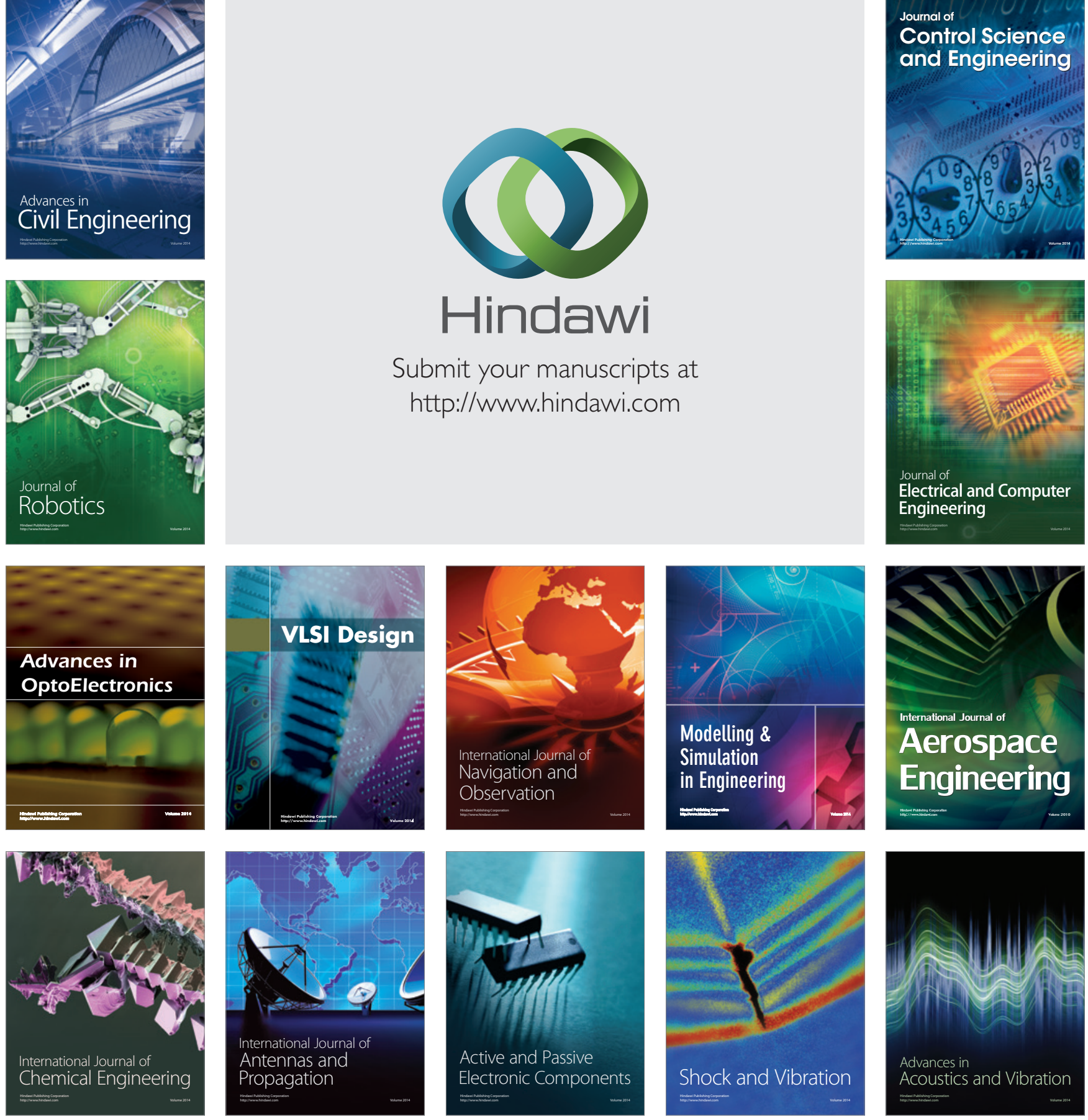